The following is a post-print of the original article:

John A. T. Norman, Melanie Perez, Stefan E. Schulz, Thomas Waechtler, "New Precursors for CVD Copper Metallization", Microelectron. Eng. 85, 2159-2163 (2008).

ISSN: 0167-9317

Digital Object Identifier: 10.1016/j.mee.2008.05.036

Available via http://www.sciencedirect.com or http://dx.doi.org/10.1016/..mee.2008.05.036

(C) 2008 Elsevier B.V.

\title{
New Precursors for CVD Copper Metallization
}

John A. T. Norman ${ }^{\mathrm{a}, *}$, Melanie Perez ${ }^{\mathrm{a}}$, Stefan E. Schulz ${ }^{\mathrm{b}}$, Thomas Waechtler ${ }^{\mathrm{b}}$

${ }^{\mathrm{a}}$ Air Products and Chemicals, 1969 Palomar Oaks Way, Carlsbad, CA 92011, USA

${ }^{\mathrm{b}}$ Chemnitz University of Technology, Center for Microtechnologies, 09126 Chemnitz, Germany

* Corresponding author. Tel.: +1 (760) 931-2033; fax: +1 (760) 929-6209. E-mail address: normanja@apci.com (J. Norman). 


\section{Abstract}

A novel CVD copper process is described using two new copper CVD precursors, KI3 and KI5, for the fabrication of IC or TSV (Through Silicon Via) copper interconnects. The highly conformal CVD copper can provide seed layers for subsequent copper electroplating or can be used to directly fabricate the interconnect in one step. These new precursors are thermally stable yet chemically reactive under CVD conditions, growing copper films of exceptionally high purity at high growth rates. Their thermal stability can allow for elevated evaporation temperatures to generate the high precursor vapor pressures needed for deep penetration into high aspect ratio TSV vias. Using formic acid vapor as a reducing gas with KI5, copper films of $>99.99$ atomic $\%$ purity were grown at $250^{\circ} \mathrm{C}$ on titanium nitride at a growth rate of

> $1500 \AA$ Amin. Using tantalum nitride coated TSV type wafers, $1700 \AA$ of highly conformal copper was grown at $225^{\circ} \mathrm{C}$ into $32 \mu \mathrm{m} \times 5 \mu \mathrm{m}$ trenches with good adhesion. With ruthenium barriers we were able to grow copper at $125^{\circ} \mathrm{C}$ at a rate of $20 \AA / \mathrm{min}$ to give a continuous $\sim 300 \AA$ copper film. In this respect, rapid low temperature CVD copper growth offers an alternative to the long cycle times associated with copper ALD which can contribute to copper agglomeration occurring.

\section{Keywords}

Copper CVD, TSV, seed layers 


\section{Introduction}

3-D packaging is steadily gaining momentum as the key technology for building high performance, energy efficient and compact semiconductor devices for a multitude of applications [1]. Processes for manufacturing the copper through silicon vias (TSVs) required for 3-D are currently under intensive investigation, including copper chemical vapor deposition (CVD). Since the latter process is well known to deposit uniform metal films with excellent step coverage, it continues to be a promising technique for semiconductor interconnect manufacturing. Being of inherently superior step coverage compared to the more line-of-sight physical vapor deposition technique (PVD), it can provide conformal seed layers for integrated circuit (IC) or TSV interconnect via fill by copper electroplating. It can also be used to fill vias and build the interconnects directly.

With PVD techniques to date being successfully applied for damascene IC metallization, PVD copper is currently under consideration for fabricating TSV seed layers. However, the relatively massive scale of TSVs hampers PVD's ability to provide conformal copper films, especially in vertical walled high aspect ratio vias. By contrast, copper CVD has been successfully demonstrated [2] as an effective alternative process for this application, the natural extension of which is complete via fill. As 3-D matures, the density of TSV structures per unit area will greatly increase, driving their aspect ratios higher. From this perspective, the evolution of TSV geometries makes copper CVD additionally attractive. Also, it can be accomplished on a cluster tool thereby offering the potential of sequentially depositing a diffusion barrier and then copper seed or fill. 
The barrier metal choice for IC interconnects continues to be a combination of $\mathrm{Ta}$ and $\mathrm{TaN}$. An ultra thin layer of ruthenium may prospectively be added as a 'glue layer' to provide good adhesion between the barrier structure and copper. For this reason, we explored depositions onto ruthenium using both KI3 and KI5 precursors, particularly at low wafer temperatures to help avoid copper agglomeration which can disrupt the continuity of the thin copper seed films. For TSVs, this is not an issue since the seed layers can be grown much thicker. Candidate TSV barrier materials include TaN, TiN and TaCN all of which were used in our study. The first two can be deposited by PVD, CVD or ALD and the last potentially by CVD or ALD.

\section{Precursor considerations}

An effective copper CVD precursor must deliver pure copper films with good step coverage and high growth rates. It must also be stable at elevated evaporation temperatures to deliver high vapor pressures for high CVD growth rates. This is especially important for TSV metallization where a high via density per unit area of wafer effectively amplifies the substrate surface to be metallized. Thus, precursor depletion near the bottom of the vias must be avoided by using high precursor vapor pressures to drive the metal deposition right to the bottom of these structures. Additionally, a wide process window is desirable to allow both the low temperature growth of ultra thin IC copper seed layers and higher temperature rapid copper growth for TSV type packaging applications.

The new molecules KI3 and KI5, originally designed for ALD copper [3] are direct descendants of the most widely used copper precursor, $\mathrm{Cu}(\mathrm{hfac})(\mathrm{tmvs})$ (i.e. CupraSelect ${ }^{\circledR}$ ) 
[4]. This fluorinated precursor grows pure copper films at a temperature of $\sim 200^{\circ} \mathrm{C}$ in a thermal disproportionation reaction according to the Equation (1) below. The organometallic bond between copper and the olefin group is weak and easily broken allowing the release of tmvs as a free molecule which drives the metallization reaction. As can be seen, for every two atoms of copper introduced into the CVD chamber as precursor, one is lost to the CVD effluent stream as the volatile by-product $\mathrm{Cu}(\mathrm{hfac})_{2}$. This sets the maximum metallization efficiency at $50 \%$ and the $\mathrm{Cu}(\mathrm{hfac})_{2}$ by-product, a solid at room temperature, needs to be managed downstream of the reactor. Copper deposition made onto barrier films such as tantalum, tantalum nitride or titanium nitride tends to exhibit poor adhesion since fluorine containing impurities from precursor decomposition form at the copper-barrier interface. However, this adhesion problem does not apply to ruthenium barriers.
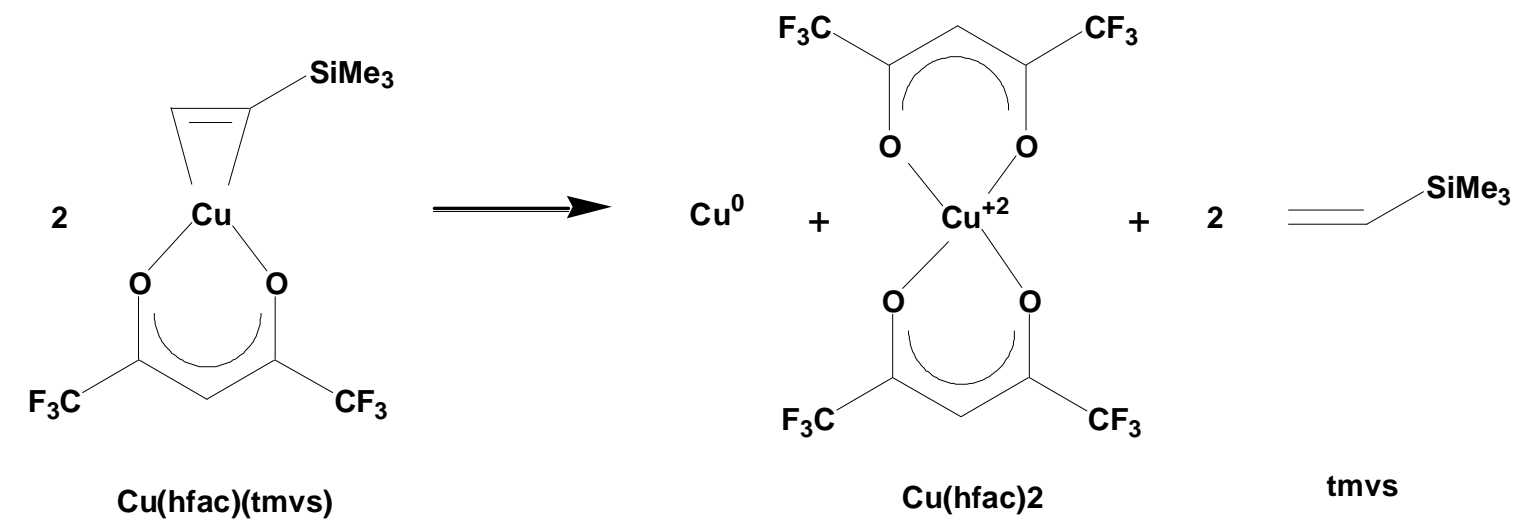

\section{Structures and thermal properties of KI3 and KI5}

In the molecules KI3 and KI5, shown in Figures 1 and 2, a similar olefin group as found in CupraSelect ${ }^{\circledR}$ is directly attached to the main body of the precursor to form a seven membered ring as it coordinates through an organometallic bond to copper. 


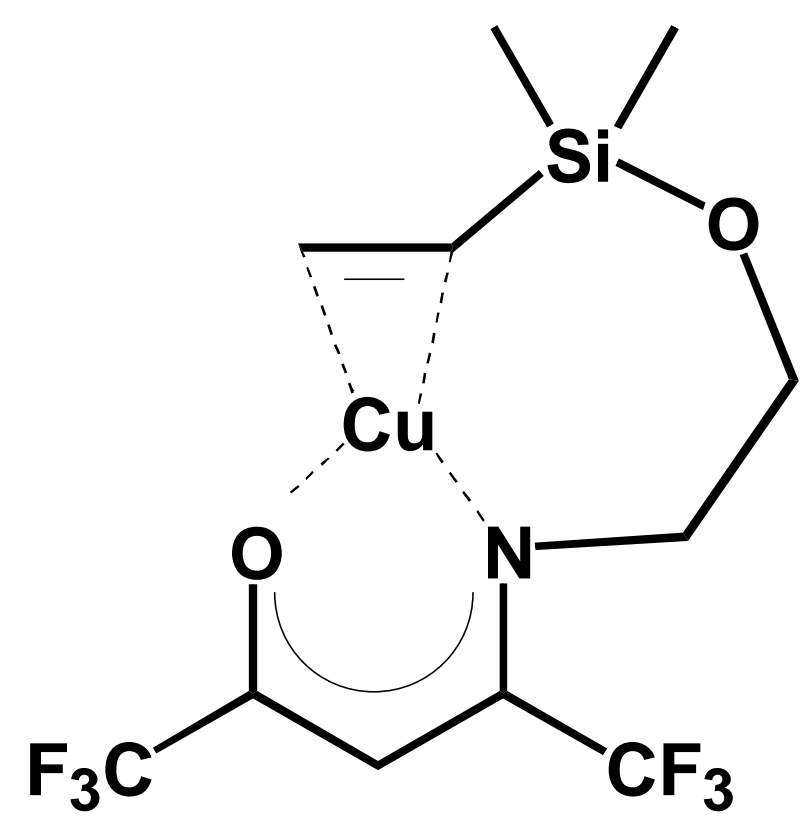

Fig. 1. Copper precursor KI3.

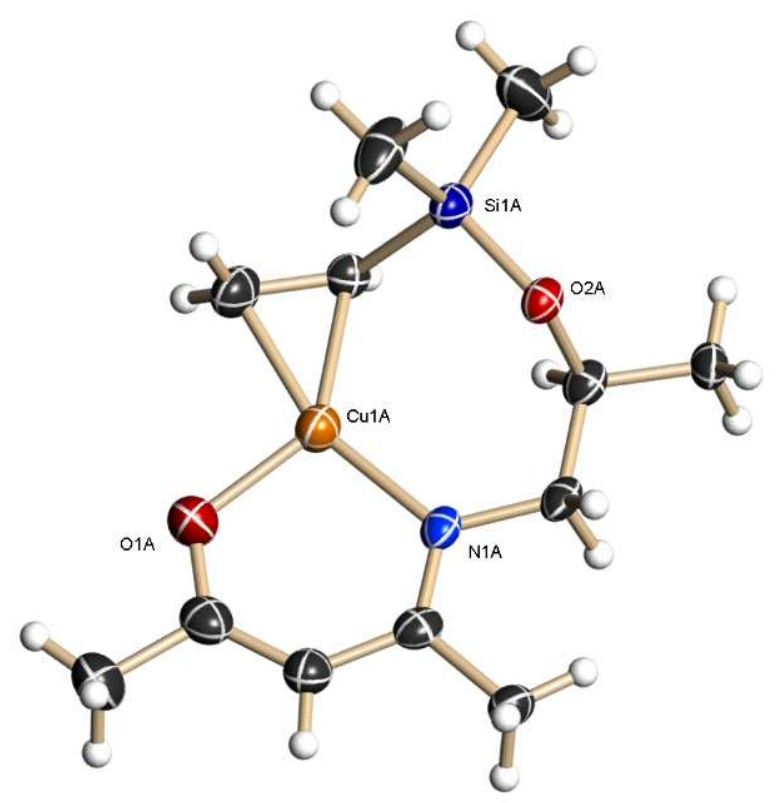

Fig. 2. Copper precursor KI5.

The formation of this ring structure imparts great stability so that heating these precursors leads to intact evaporation of the precursor rather than disproportionation or decomposition, 
as shown in the thermogravimetry (TGA) and differential scanning calorimetry (DSC) results in Figure 3. Being fluorinated, KI3 is considerably more volatile than unfluorinated KI5, as displayed in Figure 4. However, since these molecules are thermally stable, high source temperatures can be used to deliver high vapor pressures without the concern of decomposition occurring. For example, at $140^{\circ} \mathrm{C} \mathrm{KI} 5$ has a vapor pressure of $>2$ Torr which exceeds the maximum vapor pressure achievable from CupraSelect ${ }^{\circledR}$ at $\sim 70^{\circ} \mathrm{C}$ since above this temperature the latter can decompose. KI5 also avoids both fluorine based adhesion problems and the cost associated with fluorinated precursors. Having established the stability and volatility of these molecules, our challenge in this work was to then find a way to use them as precursors for copper CVD, focusing mostly on KI5.

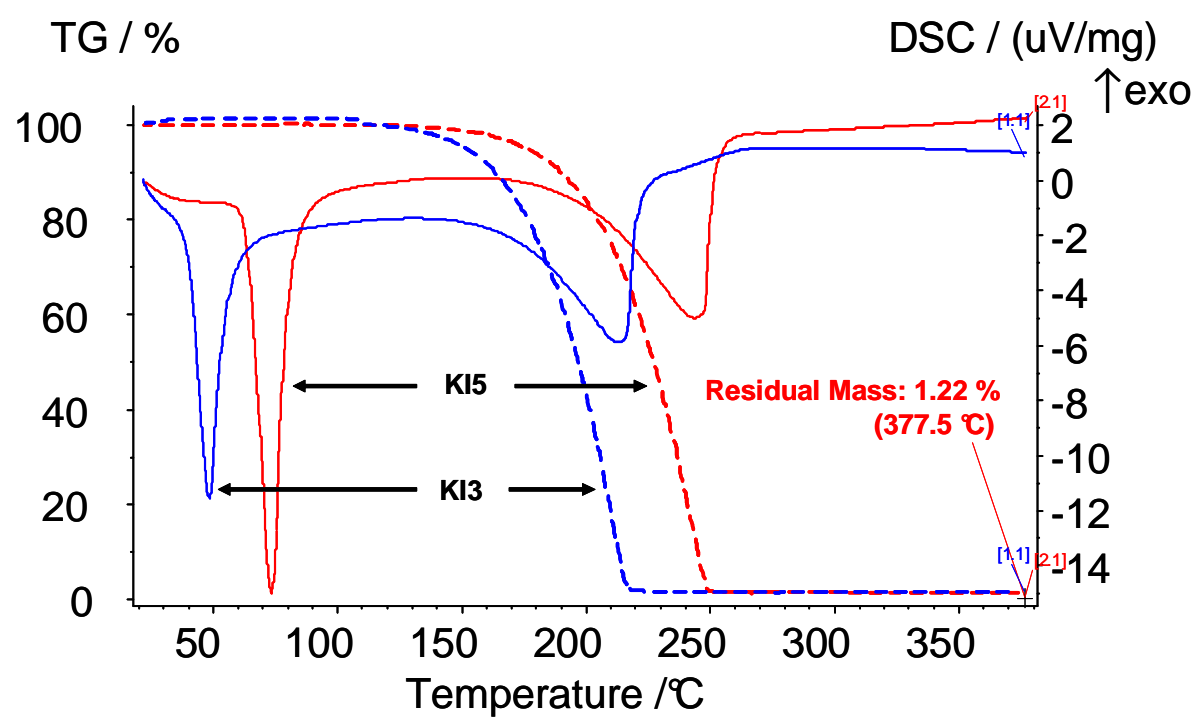

Fig. 3. TGA (dashed line) and DSC (solid line) for KI3 and KI5. 


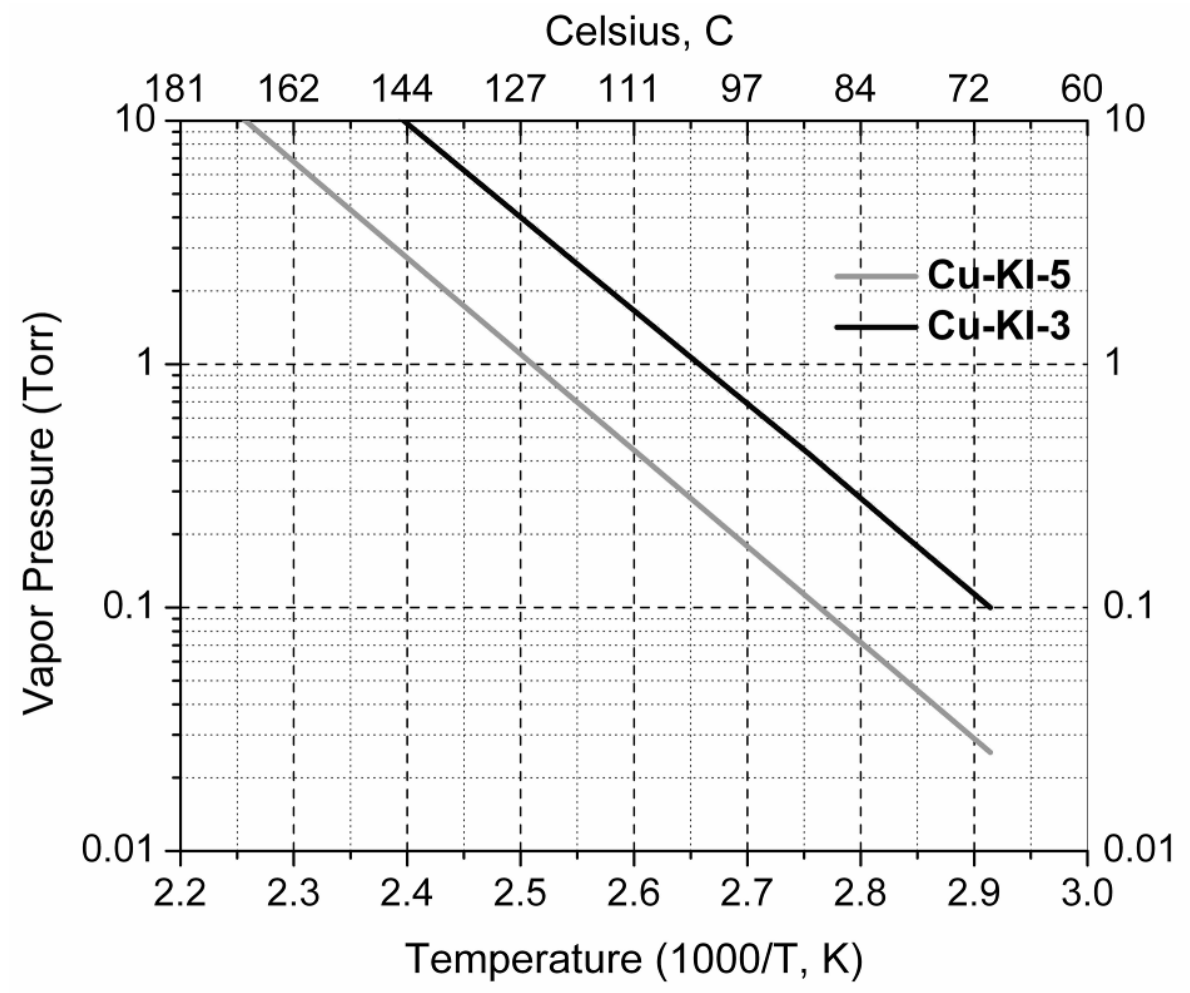

Fig. 4. Vapor pressures of KI3 and KI5.

\section{Screening KI3 and KI5 as CVD copper precursors}

\subsection{KI3}

We started our evaluation with the $\mathrm{KI} 3$ precursor and then applied our findings towards building a CVD process using KI5. For these purposes we used both a home built hot wall reactor and a modified Varian Gartek single wafer reactor $[5,6]$. In the former, the KI3 was delivered into the system by simple bubbling whereas in the latter the KI3 was dissolved in $\mathrm{N}$-methylpyrrolidone (NMP) at 3:1 KI3 to NMP weight ratio. The resulting solution was dosed into the reactor by a Bronkhorst liquid delivery system (LDS). In this approach the precursor stock is kept at room temperature. Only during the CVD process is the liquid 
transported to an evaporator unit where it is vaporized at 85 to $100^{\circ} \mathrm{C}$ and mixed with argon carrier gas before it is introduced into the reactor. The net results of these experiments were that hydrogen, water and isopropanol, and mixtures thereof, would only give scattered copper nuclei on titanium nitride and ruthenium substrates (although favoring ruthenium) when using the Gartek reactor and either faint or no copper depositions using the hot wall reactor with hydrogen. This was a surprise, since previously we had successfully run KI3 in ALD mode using hydrogen in the same temperature range [7]. However, when we tried formic acid as reagent gas in either reactor we readily achieved copper deposition, presumably through the reducing action of the atomic hydrogen which forms when formic acid contacts various metal surfaces, including copper and ruthenium [8]. Alternatively, the formic acid and copper precursor form a complex which then breaks down to give copper metal.

\subsection{KI5}

We then ran $\mathrm{KI} 5 /$ formic acid, under comparable conditions to $\mathrm{KI} 3$, in the hot wall reactor where at $250^{\circ} \mathrm{C}$ we grew $3.5 \mu \mathrm{m}$ of copper onto titanium nitride at a rate of $>1500 \AA / \mathrm{min}$ and showed by SIMS analysis that the copper contained no detectable carbon, hydrogen, nitrogen, oxygen or silicon making it at least 99.99 atomic\% copper. We also achieved deposition of $300 \AA$ pure and continuous copper on ruthenium at $125^{\circ} \mathrm{C}$, at a rate of $20 \AA / \mathrm{min}$ as shown in Figure 5. At this point we then transferred the formic acid process to a Vactronics [9] single wafer reactor to more carefully explore the properties of KI5. A summary of conditions used in the screening studies are found in Table 1. 


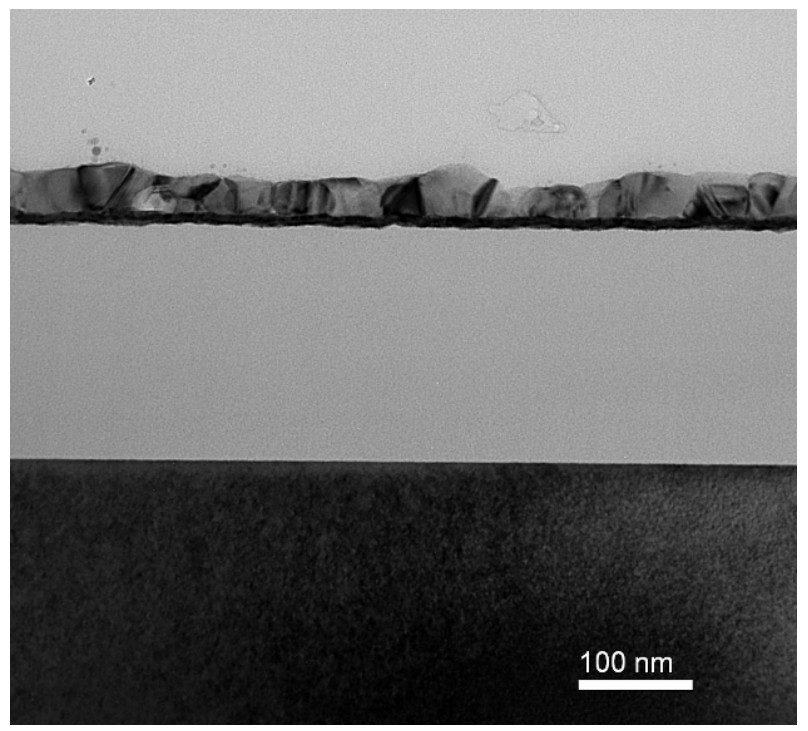

Fig. 5. A TEM cross section of $\sim 300 \AA$ of copper grown onto $\mathrm{Ru}$ at $125^{\circ} \mathrm{C}$ using $\mathrm{KI} 5 /$ formic acid in the hot wall reactor.

Table 1. Basic CVD process conditions for the Gartek single wafer and hot wall reactors used in the screening studies.

\begin{tabular}{|c|c|c|c|c|c|c|}
\hline Reactor & $\begin{array}{l}\text { Chamber } \\
\text { Pressure } \\
\text { (Torr) }\end{array}$ & $\begin{array}{l}\text { Wafer temp } \\
\text { range }\left({ }^{\circ} \mathrm{C}\right)\end{array}$ & $\begin{array}{l}\text { Formic acid } \\
\text { feed rate }\end{array}$ & $\begin{array}{c}\text { Precursor } \\
\text { source }\left({ }^{\circ} \mathrm{C}\right)\end{array}$ & $\begin{array}{l}\text { Precursor } \\
\text { carrier gas } \\
\text { flow rate } \\
(\text { sccm) } \\
\end{array}$ & $\begin{array}{l}\text { KI3 feed } \\
\text { rate with } \\
\text { LDS } \\
\text { (mg/min) }\end{array}$ \\
\hline $\begin{array}{l}\text { Hot wall } \\
\text { (KI3) }\end{array}$ & 2 & $150-250$ & $150 \mathrm{sccm}$ & $57-75$ & 25 & \\
\hline $\begin{array}{l}\text { Single wafer } \\
\qquad(\mathrm{KI} 3)\end{array}$ & 1 & $230-300$ & $82 \mathrm{mg} / \mathrm{min}$ & 100 & 500 & 41 \\
\hline $\begin{array}{l}\text { Hot wall } \\
\text { (KI5) }\end{array}$ & 2 & $125-250$ & $150 \mathrm{sccm}$ & 85 to 120 & 25 & \\
\hline
\end{tabular}




\section{CVD copper processing using the Vactronics reactor}

\subsection{TiN, TaN and TaCN substrates}

Figure 6 shows the copper film thickness as a function of run time using TaN, TiN and TaCN substrates. The KI5 source was kept at $120^{\circ} \mathrm{C}$, using $100 \mathrm{sccm}$ helium as carrier gas. Formic acid reagent was delivered by vapor draw at a rate of $\sim 180 \mathrm{sccm}$ at $30^{\circ} \mathrm{C}$. The wafer temperature was $225^{\circ} \mathrm{C}$ and CVD chamber pressure was kept at 2 Torr. All three substrates show a fairly linear growth rate with time, especially for TaCN.

\section{Copper CVD using KI5/formic acid at $225^{\circ} \mathrm{C}$}

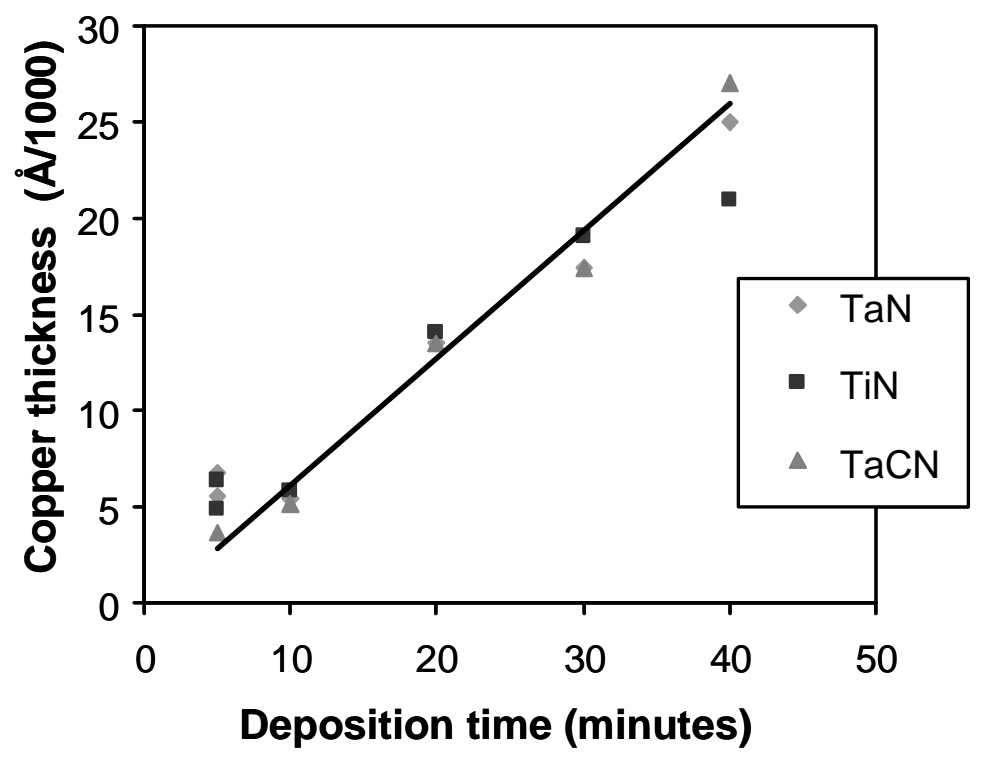

Fig. 6. Thickness of CVD copper obtained using KI5/formic acid. 


\section{Adhesion}

Excellent adhesion was observed for all copper films grown onto ruthenium up to $2.5 \mu \mathrm{m}$ thick as determined by scribing through the copper down to silicon in a \# design then applying and removing Scotch tape. No copper was removed. Similarly, adhesion was also excellent on gold and platinum i. e. metals with no native oxide thereby allowing metal to copper bonding. For $\mathrm{TiN}, \mathrm{TaCN}$, and $\mathrm{TaN}$, adhesion passed the \# Scotch tape test at

$<1800 \AA \mathrm{Cu}$, but failed at greater copper thicknesses. The TaN samples > $1800 \AA$ were annealed at $375^{\circ} \mathrm{C}$ under vacuum for $30 \mathrm{~min}$ after which they passed the adhesion test. $3500 \AA$ copper films grown onto thermal silicon dioxide at $250^{\circ} \mathrm{C}$ gave a resistivity of $2.03 \mu \Omega \mathrm{cm}$, as derived from sheet resistance measurements using a 4-point probe.

\section{TSV metallization}

We processed PVD TaN coated patterned wafers featuring trenches and vias whose dimensions approach those encountered in TSVs. Cross sectioned samples for imaging were then made by simply fracturing the wafer. The SEM images in Figure 7 show an approximately $1700 \AA$ conformal copper film grown at $225^{\circ} \mathrm{C}, 8$ Torr chamber pressure, deposited into $\sim 5 \mu \mathrm{m} \times 32 \mu \mathrm{m}$ trenches $($ aspect ratio $=6.4$ ). This result shows a high potential for our KI5/formic acid process for TSV copper metallization. 

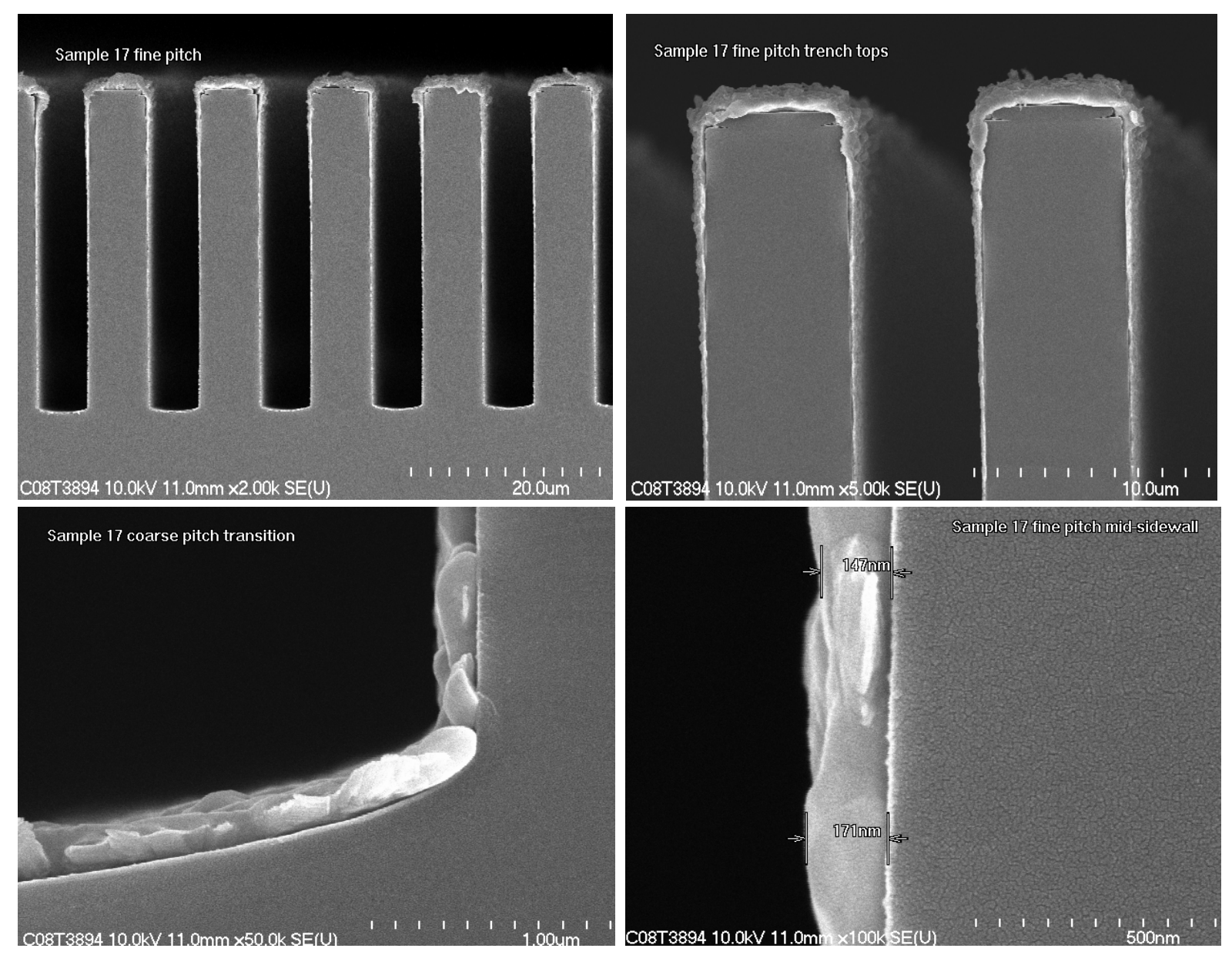

Fig. 7. Conformal copper deposition onto a TaN TSV wafer. From the top left, clockwise, is shown a row of trenches, the top of the trenches, midway on the trench wall and bottom of the trench.

\section{Summary}

For the first time, copper CVD was demonstrated using two newly developed $\mathrm{Cu}(\mathrm{I})$ ketoiminate precursors, $\mathrm{KI} 3$ and $\mathrm{KI}$. Compared to $\mathrm{Cu}(\mathrm{hfac})(\mathrm{tmvs})$ [CupraSelect ${ }^{\circledR}$ ], the established precursors for copper CVD, these two novel molecules display enhanced thermal stability while retaining high CVD reactivity. These properties allow for much higher evaporation temperatures and hence increased precursor feed rate due to enhanced vapor 
pressure both for the fluorinated KI3 and the unfluorinated KI5. With these two precursors, pure copper films were obtained using formic acid vapor as reducing gas on $\mathrm{Ru}$ and $\mathrm{TiN}$ substrates with growth rates up to $1500 \AA / \mathrm{min}\left(250^{\circ} \mathrm{C}\right.$, $\mathrm{KI} 5$ on $\left.\mathrm{TiN}\right)$. KI5 was also applied to TaN and TaCN substrates in addition to TaN TSV patterned wafers which showed a promising conformal deposition of approximately $1500 \AA$ of a copper seed layer for subsequent electrochemical metallization.

\section{Acknowledgments}

Financial support obtained from the German Research Foundation (DFG) within the International Research Training Group 1215 "Materials and Concepts for Advanced Interconnects" is gratefully acknowledged.

\section{References}

[1] A. Keigler, K. O'Donnell, Z. Liu, B. Wu, J. Trezza, Semiconductor International 30 (9) (2007) 36-44.

[2] L. Hofmann, M. Kuechler, T. Gumprecht, R. Ecke, S.E. Schulz, T. Gessner, Advanced Metallization Conference (AMC), Albany, New York USA, 2007.

[3] J.A.T. Norman, M. Perez, X. Lei, H. Cheng, ECS Transcations 3 (15) (2007) 161-170.

[4] J.A.T. Norman, B.A. Muratore, P.N. Dyer, D.A. Roberts, A.K. Hochberg, Journal de Physique I 1 (C2) (1991) 271-278.

[5] J. Röber, C. Kaufmann, T. Gessner, Applied Surface Science 91 (1995) 134-138. 
[6] H. Wolf, J. Röber, S. Riedel, R. Streiter, T. Gessner, Microelectronic Engineering 45 (1999) 15-27.

[7] J. Norman, M. Perez, AVS 7th International Conference on Atomic Layer Deposition (ALD 2007) San Diego, CA (USA) (2007).

[8] Y.-K. Sun, W.H. Weinberg, Journal of Chemical Physics 94 (6) (1991) 4587-4599.

[9] J.A.T. Norman, D.A. Roberts, A.K. Hochberg, Materials Research Society Symposium Proceedings 282 (1993) 347-352. 